\title{
Work-related musculoskeletal disorders in
}

\section{sonographers: a review of causes and types of injury and best practices for reducing injury risk}

This article was published in the following Dove Press journal:

Reports in Medical Imaging

15 February 2014

Number of times this article has been viewed

\section{Carolyn T Coffin}

Department of Diagnostic Ultrasound, Seattle University, Seattle, WA, USA

\begin{abstract}
Work-related musculoskeletal disorders in sonography professionals have a reported incidence of $90 \%$. These disorders are defined as conditions that are either caused by or aggravated by tasks performed in the workplace. These injuries have a financial and emotional impact on the worker and affect workplace productivity and quality patient care. The causes for these injuries are multifactorial and therefore require a variety of solutions for mitigating injury risk. Sonographer work postures, work schedules, task rotation, administrative support, and ergonomic workplace equipment all enter into the formula for reducing the incidence of these disorders.
\end{abstract}

Keywords: work-related musculoskeletal disorders, WRMSD, best practices, workstation, bedside studies, ergonomics, sonography

\section{Introduction}

Many professions have documented work-related musculoskeletal disorders (WRMSDs) for a number of years, and those industries have attempted to address injury risks. These injuries are defined as conditions that are caused by or aggravated by workplace activities, and they account for up to $60 \%$ of all workplace illnesses. ${ }^{1}$ They are known by different names, such as repetitive strain injury, repetitive motion injury, and cumulative trauma disorder.

Injury among sonographers was first reported in 1985 when Craig $^{2}$ identified “sonographer's shoulder." WRMSDs were first identified in cardiac sonographers in 1993 and were more extensively reported in surveys conducted in 1995 and 2008. ${ }^{3-6}$ There are multiple causative factors for these injuries; therefore, preventing them requires various strategies.

\section{Incidence of injury}

The incidence of musculoskeletal injury among North American sonographers in 1997 was $84 \%$ and had increased to $90 \%$ in $2008.4,5$ This latter result may be due to an increased awareness among sonographers as to the reason for their fatigue and pain during the workday and to a willingness to report their symptoms.

\section{Causes for injury}

The causes and contributory factors of these injuries in sonographers are numerous, but they can generally be grouped into three categories.

1. Biomechanical/environmental factors: these include the workstation, the exam room equipment, the ultrasound system, and the exam room layout. In an effort to
Department of Diagnostic Ultrasound Seattle University, 90I I2th Avenue, Seattle, WA 98I22, USA

Email coffinc@seattleu.edu 
increase throughput and standardize exams, ultrasound systems have added protocol management software that can potentially reduce exam time and increase the number of exams done by each sonographer.

2. Administrative factors: these relate to scheduling of patient exams and sonographer rotation schedules. Work conditions have changed. Employees may have to work beyond their regularly scheduled hours due to staffing shortages or busier patient schedules, which leads to reduced muscle recovery time. Rapid technological changes have resulted in redefining the nature of many jobs. ${ }^{7}$ Staffing shortages and decreased reimbursements have contributed to increased patient volumes. In addition, there is a trend toward performing ultrasound exams ordered on inpatients at their bedside rather than transporting the patients to the ultrasound department. Bedside exams present many ergonomic challenges that are difficult to address.

3. Worker practices: these are the postures sonographers use when scanning or working at a computer workstation. In addition, job satisfaction can play a role in these injuries. Pressures and stress in the workplace have been identified as potentially affecting worker health. ${ }^{7}$ Automated work practices often leave employees with the feeling that they have little control over their workload. ${ }^{7}$ Although job satisfaction has been more strongly correlated with psychosocial problems than with physical illness, there is some correlation between job satisfaction and musculoskeletal disorders.

Other contributory factors are the aging workforce and increasing obesity rates. ${ }^{8}$ The average age of sonographers in the 2008 survey was 45 years. ${ }^{5}$ The impact of age is related to muscle force. ${ }^{9}$ By age 45 , both males and females are approximately 10 years beyond their peak muscle strength, as maximum muscle strength is reached in the second and third decades. ${ }^{9}$ Obese patients require the sonographers to exert more force during the ultrasound exam and may require an extended reach and/or excessive arm abduction to access both sides of the patient. Obesity also puts workers at risk for health conditions that can make them more susceptible to WRMSDs.

\section{High-risk work activities}

According to the Occupational Safety and Health Administration, the work activities that can lead to WRMSDs are repetitive motions, forceful or awkward movements, duration of pressure, overuse, poor posture or improper positioning, excessive force and strain, and vibrations. ${ }^{10}$
All but vibrations are work activities engaged in by sonographers.

Repetitive motions occur when sonographers perform the same type of exam throughout the workday. This is more prevalent among sonographers performing high-risk obstetrical ultrasound exams and cardiac sonographers. The lack of variety in these exams forces the sonographers to use the same muscle activities all day without enough recovery time. The duration of pressure is related to the amount of time that the sonographers are actually scanning, or "transducer time." This is also related to the amount of transducer pressure being exerted by the worker while scanning. Poor posture and improper positioning of extremities include wrist flexion and extension, neck twisting or extension, trunk twisting and flexion, reaching, and arm abduction (Figures 1-5). Static work postures reduce blood flow to the joints, which then increases the load on the muscles and decreases the time for fatigue to occur.

\section{Injury symptoms and the impact of injury}

WRMSD symptoms include inflammation and swelling, numbness, muscle spasm, burning and/or tingling, and loss of sensation. Some of these symptoms may cause a loss of muscle strength, making it difficult to hold a transducer. ${ }^{4-6,11}$ Eye strain, although not a symptom of WRMSD itself, can lead to blurred vision and poor posture. As this is a cumulative disorder, seemingly minor symptoms can progress over time to more serious, even debilitating, symptoms.

Many of the challenges facing imaging departments center around a stable, experienced workforce, which means that worker injury can have a large impact on the

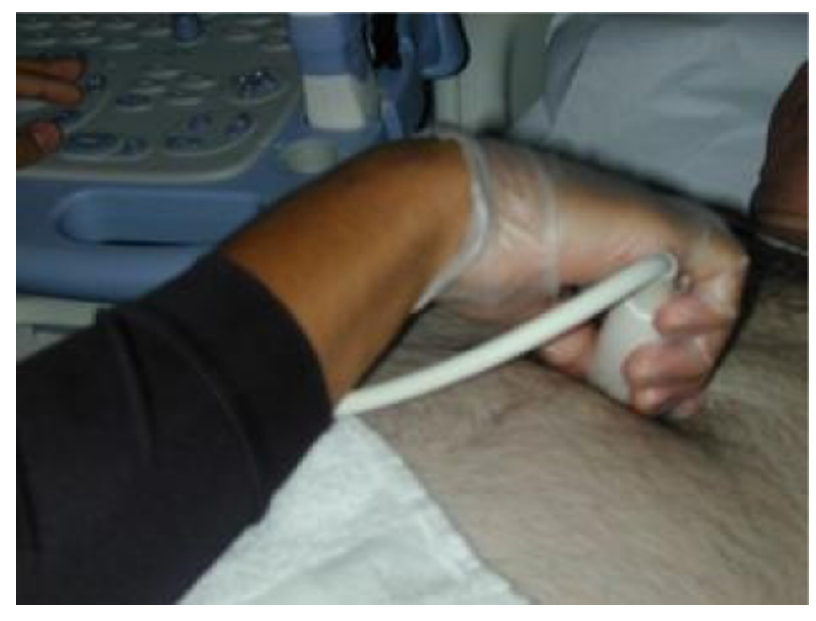

Figure I Wrist flexion and "pinch" grip. 


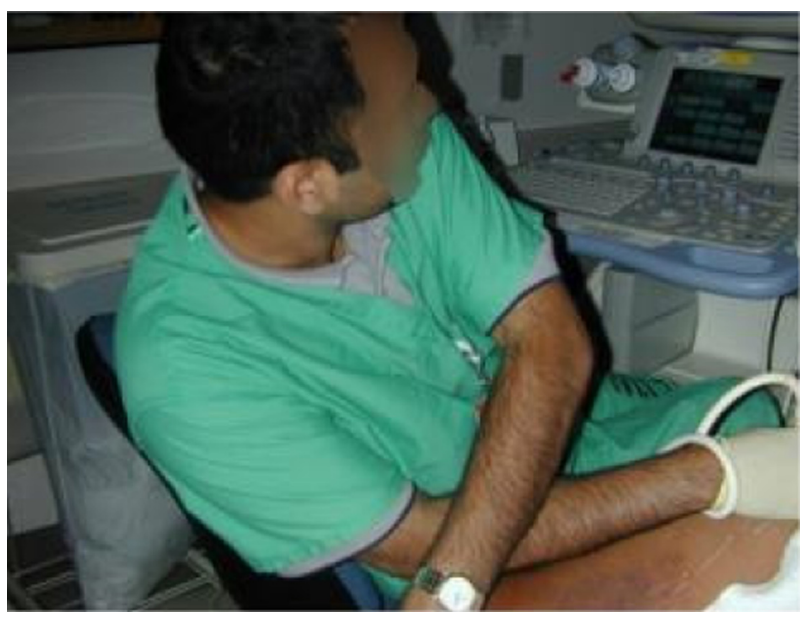

Figure 2 Trunk and neck twist.

department's bottom line, worker morale, and the quality of patient care. There is also a financial and emotional impact on the individual worker who becomes injured. Increasing an ultrasound department's work flow and/or staffing reductions could potentially result in each sonographer performing more exams each day. This increased volume could lead to injuries if the individual worker is unaware of how to optimize the workstation and how to make changes in his/ her work postures.

\section{Types of injury}

Sonographers are at risk for the following injuries.

- The shoulder is the most common body part injured in sonographers, and the injuries include bursitis, tendonitis, and rotator cuff tears. ${ }^{4,5}$ The rotator cuff muscles and their tendons are poorly perfused normally, and any work activities that further compromise blood flow

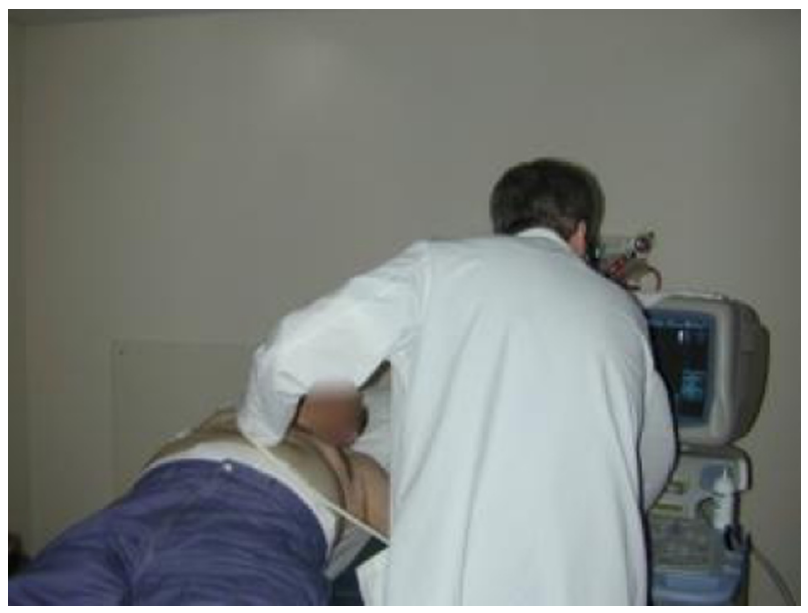

Figure 3 Trunk flexion.

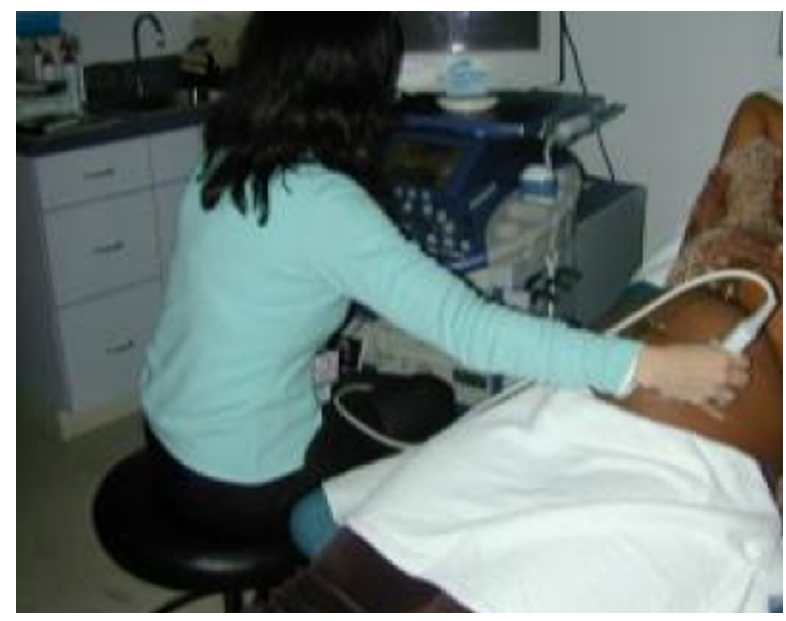

Figure 4 Arm abduction.

to the shoulder can decrease the inflow of oxygen and nutrients and the removal of wastes. This, in turn, can increase the risk for rotator cuff injury. As a person ages, he/she is at higher risk for rotator cuff injury simply as a factor of aging. Thus, as the workforce continues to age, this injury may become more prevalent in occupations that require prolonged arm abduction and static arm postures.

- Inflammation of the tendons (tendonitis) and/or tendon sheath (tenosynovitis) of the hand and wrist are also common among sonographers. ${ }^{11}$ Gripping the ultrasound transducer contributes to this injury and can lead to "trigger finger," which occurs when the tendon sheath becomes edematous and traps the finger tendon in a locked position. ${ }^{4,6,12-14}$ Another form of tendonitis is De Quervain syndrome, which occurs at the base of the thumb and is also associated with gripping the ultrasound transducer

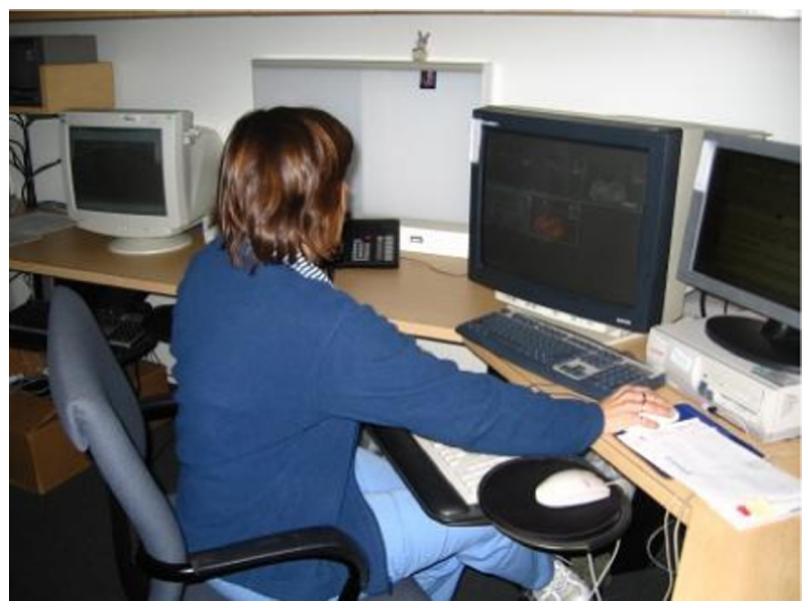

Figure 5 Excessive reaching. 
and/or pressure on the base of the thumb against the transducer casing. ${ }^{15}$

- Nerve entrapment syndromes, such as carpal tunnel syndrome, cubital syndrome, and, to a lesser extent, thoracic outlet syndrome occur in sonographers and affect not only muscles and tendons but also nerves and the circulation to the arm and hand. It has been demonstrated that carpal tunnel syndrome results from activities that involve flexion and extension of the hand, which increases pressure on the median nerve in the wrist. ${ }^{16}$ In sonography, wrist flexion and extension results from gripping and manipulating the ultrasound transducer during a variety of exams. Cubital tunnel syndrome can result from repeated flexion and extension of the elbow or from mechanical pressure against the ulnar nerve when a sonographer rests his/her elbow on the exam table while scanning. ${ }^{13}$

\section{Muscle physiology}

An understanding of basic muscle physiology and the importance of optimal body mechanics and neutral work postures will help sonographers identify and reduce the risk factors associated with their profession. There are two types of muscle activity: dynamic and static. When performing dynamic work, the contraction and relaxation activity of the muscles flushes them with oxygen-rich blood and allows for the removal of waste products. Static work postures, on the other hand, prevent the muscles from receiving fresh blood and lead to a buildup of waste products. Muscles must rely on their own reserves, and they eventually become fatigued. ${ }^{17}$ The onset of muscular fatigue from static effort will be more rapid with the exertion of greater force. Research has shown that work can be maintained for several hours per day without symptoms of fatigue if the force exerted does not exceed approximately $10 \%$ of the maximum force of the muscle involved. ${ }^{18-20}$ When the frequency and duration of loading exceeds the ability of the muscles and tendons to adapt, inflammation occurs, followed by degeneration, microtears, and scar formation. ${ }^{15,18}$ Muscles and tendons are designed to stretch and to be used regularly; therefore, an important injury prevention strategy is movement.

\section{High-risk work postures}

A common static work posture among sonographers is abduction of either the scanning arm or the nonscanning arm. An angle of abduction greater than $30^{\circ}$ puts the shoulder at risk for injury, especially if the arm is abducted for long periods of time. ${ }^{17,21}$ Sonographers often scan with excessive arm abduction because they do not take the time to optimize their position in respect of the patient and the ultrasound system. Over-reaching is another common injury-producing work posture. Reaching with either arm beyond $30 \mathrm{~cm}$ for a prolonged period of time can result in muscle fatigue and possible injury. ${ }^{17}$ Reach and abduction are both exam specific and depend on a patient's body habitus and on the adjustability of exam room equipment. However, clinical site work evaluations have demonstrated that the amount of reach for a sonographer to scan the left side of a patient's abdomen or the left lower extremity can be as much as $63 \mathrm{~cm}$. For cardiac sonographers who scan from the right side and reach over the patient, the amount of reach can exceed $71 \mathrm{~cm}$. Right-handed cardiac sonographers also typically have too much arm abduction and too much wrist flexion and trunk bending, as they are reaching over the patient to access the cardiac apex for a majority of the exam (Figure 6).

Forward reach to the ultrasound control panel varies depending on which controls are being accessed and the design and depth of the ultrasound system's control panel. Full arm extension may be required to reach the most distant controls; however, this is a high-risk posture only if the sonographer maintains this reach for an extended period of time. Typically, sonographers reach for controls and then relax the nonscanning arm. When they rest their nonscanning arm on the ultrasound system at a distance in excess of $30 \mathrm{~cm}$, the risk for injury increases.

Injury-producing work postures are especially common when performing venous reflux exams. Typically, these are performed with the patient standing, either on the floor or on a step stool, which forces the sonographers to sit or kneel on the floor (Figure 7). The resultant work postures are excessive reach, particularly with the nonscanning arm, excessive neck extension, and trunk twist.

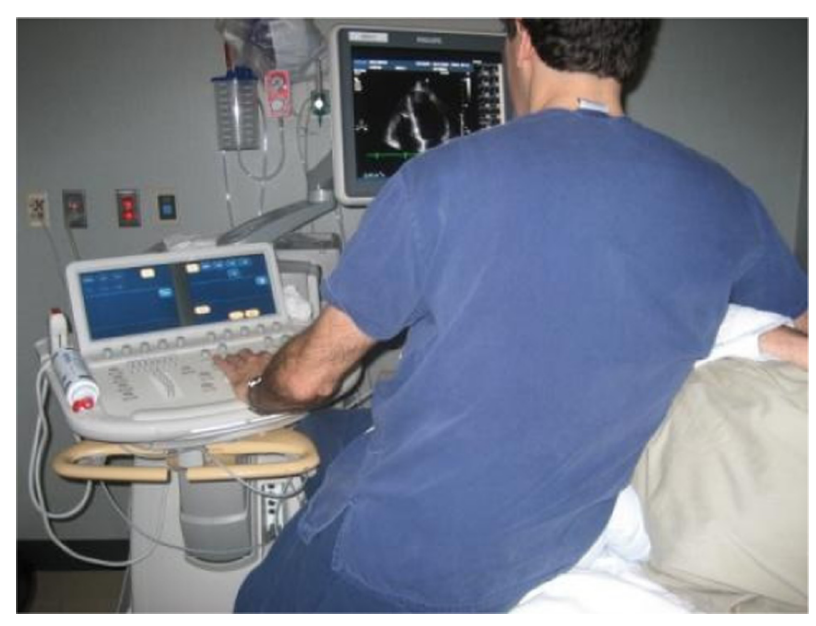

Figure 6 Right-handed cardiac sonographer demonstrating too much arm abduction, reach, trunk bending, and neck flexion. 


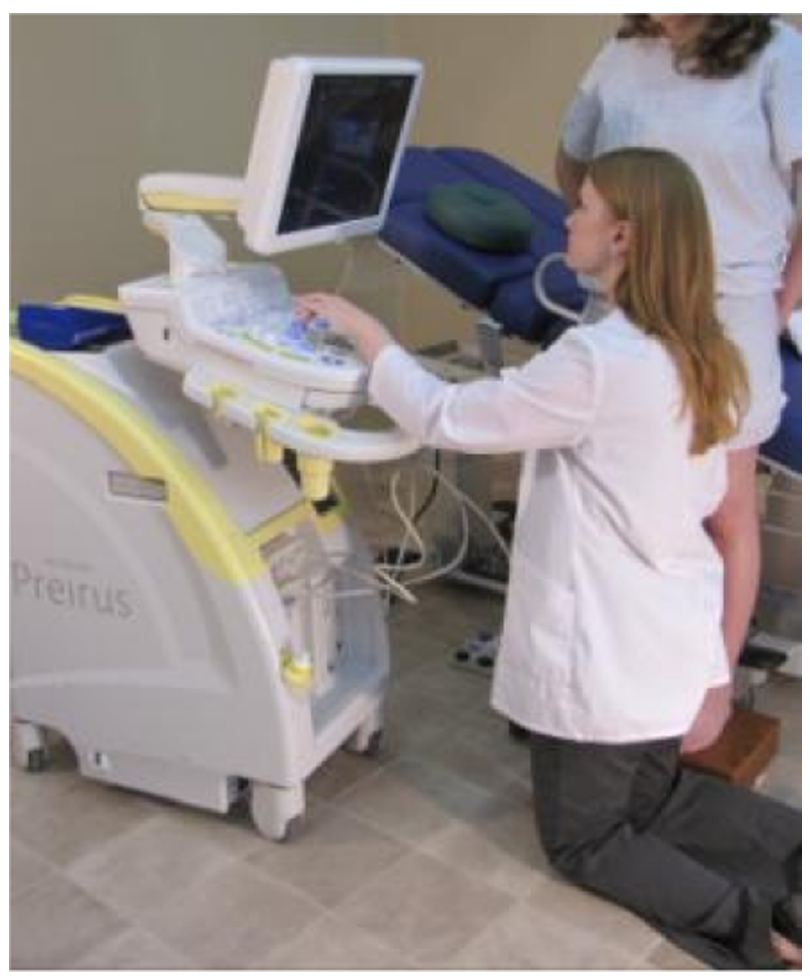

Figure 7 Kneeling on the floor to perform venous reflux study.

In addition to abduction and over-reaching, performing exams with the scanning arm behind the shoulder (Figure 8), reaching across the front of the body to access the control panel, and scanning with the arm unsupported can all put strain on the shoulder joint.

Transducer designs can often force the use of a "pinch" grip rather than a whole-hand or palmar grip. As ultrasound manufacturers cannot produce a transducer for each individual user, sonographers must learn to hold the transducer in a more comfortable grip. A pinch grip uses the smallest

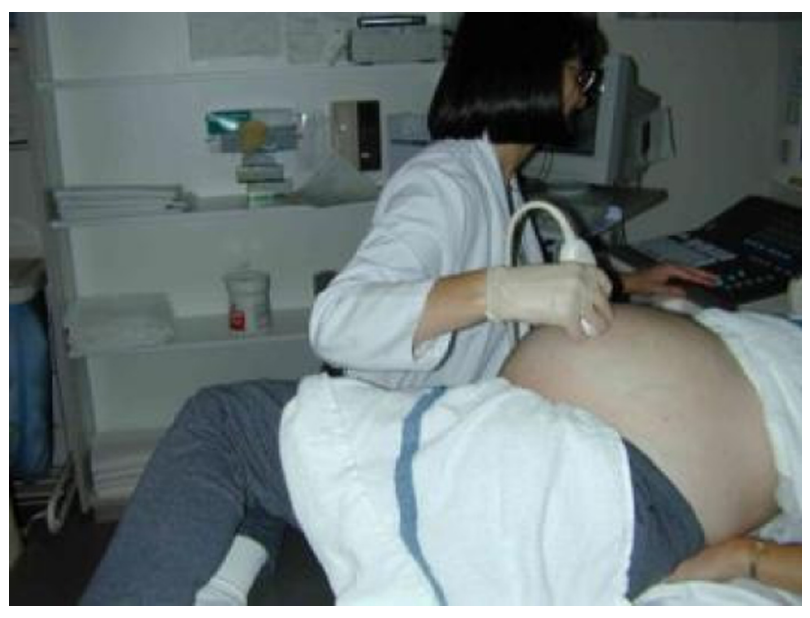

Figure 8 Scanning with arm behind the shoulder. muscles of the body and requires up to five times more muscle and tendon force than a palmar grip. ${ }^{22}$ Therefore, sonographers should try different ways of holding the transducer that use more of the hand-grips similar to those used to hold golf clubs or baseball bats. The three-dimensional/ four-dimensional transducers are wider and heavier than two-dimensional transducers, contributing to the discomfort of gripping them. Manufacturers have varied the design of transducers, including a fingertip transducer and a prototype of a wireless transducer. However, these are not widely available for all ultrasound systems, nor are they appropriate for all types of exams and procedures. In addition, wireless transducers may add extra weight to the sonographer's hand and wrist, as those transducers have to accommodate a battery pack. Attempts have been made to design a "sleeve" that can slide over the narrow transducers to make them more comfortable. However, these are generally just one size, can be awkward, and may be easily lost in the department.

\section{Best practices for mitigating sonographer occupational injury}

As these injuries have multiple causative factors, reducing or eliminating injury risk hazards can be done in multiple ways. Preventive measures can range from simple work posture changes that conform to the department's existing equipment to upgrading the department with state-of-the-art ultrasound systems, exam tables, and chairs. However, all the best equipment is only as good as the willingness of the user to optimize the features of that equipment and to make work posture changes. Therefore, it is a combination of factors that has a positive impact on reducing injury risk hazards.

Sonographers should sit or stand so that they can move down the length of the exam table when necessary to prevent reaching back while scanning. They should also position themselves in front of the most frequently used keys on the ultrasound control panel to avoid reaching across their bodies. Strain on the shoulder can be reduced by using an arm support device while scanning.

Both arm abduction and over-reaching can be reduced by lowering the exam table and/or raising the chair, by having the patient move close to the sonographer, and by positioning the ultrasound system's control panel close to the sonographer (Figure 9). A qualitative study looked at the reduction in muscle firing when the scanning arm was supported. This was evaluated using surface electromyography (SEMG) that provided audible feedback. Muscle firing, as determined by the beeping of the SEMG unit, was significantly reduced when using the arm support device seen in Figure $10 .{ }^{23}$ Another study using 


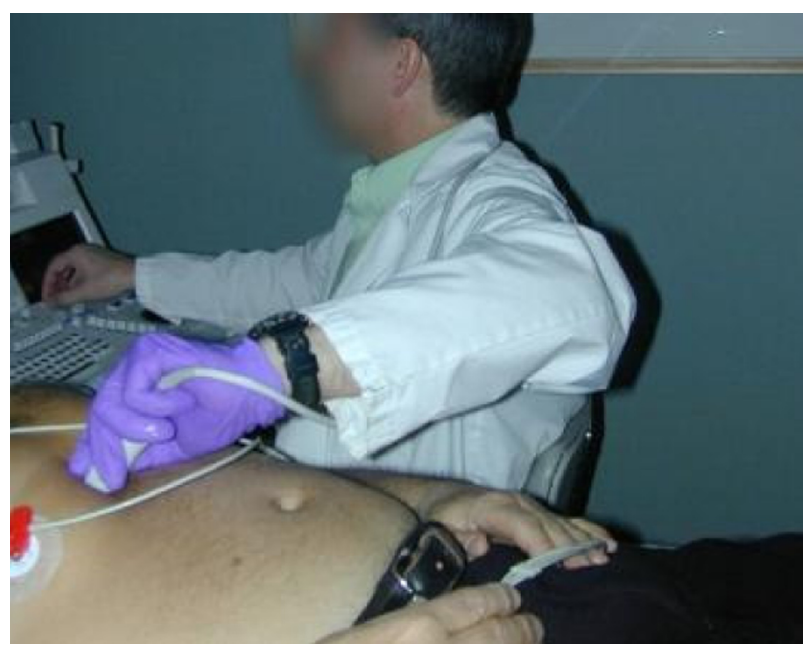

Figure 9 Left-handed cardiac exam; chair is too low or table is too high.

SEMG to measure muscle firing used by sonographers in the "typical" scanning posture demonstrated a $46 \%$ reduction in muscle firing when the angle of abduction was reduced from $75^{\circ}$ to $30^{\circ}$. When the reduced angle of abduction was supplemented with support cushions for the scanning arm, there was an $88 \%$ reduction in muscle firing (Figure 11). In addi-

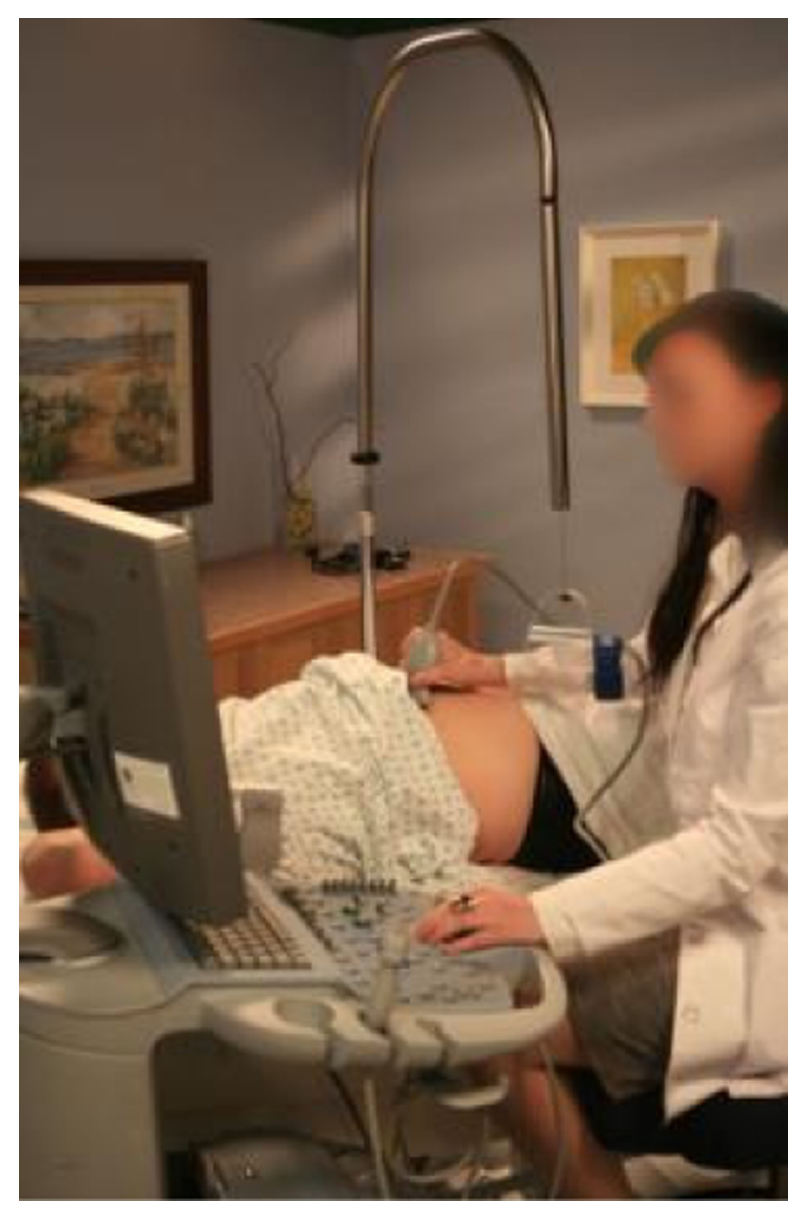

Figure 10 Mobile arm support device.

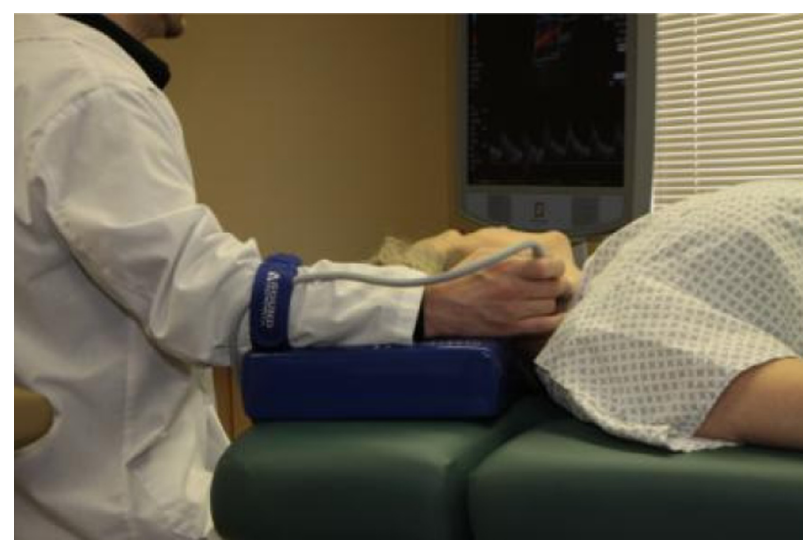

Figure I I Arm support cushion.

tion, the study demonstrated a $64 \%$ reduction in firing of the left trapezius muscle when the reach to the control panel was reduced to $30 \mathrm{~cm}^{24}$

When scanning the left side of a patient's abdomen, the sonographer should have the patient roll up on his or her side and move to the edge of the exam table toward the sonographer. When performing endovaginal exams, the sonographers should use exam tables with stirrups, if available, and scan from the foot of the table (Figure 12). If the table does not have stirrups, sonographers can still have the patient move to the end of the exam table and place her heels on each corner of the table. This will enable the sonographers to scan from the end of the table.

When scanning the patient's neck for either carotid artery exams or thyroid exams, the sonographer should scan from the head of the table, facing the patient's feet (Figure 13). However, if sonographers find this position too challenging, they should sit or stand level with the patient's shoulder, and when scanning the left side have the patient lie at an angle with his/her head as close to the sonographer as possible. They should also try having the patient sit for these exams and then stand behind him/her (Figure 14). This allows the

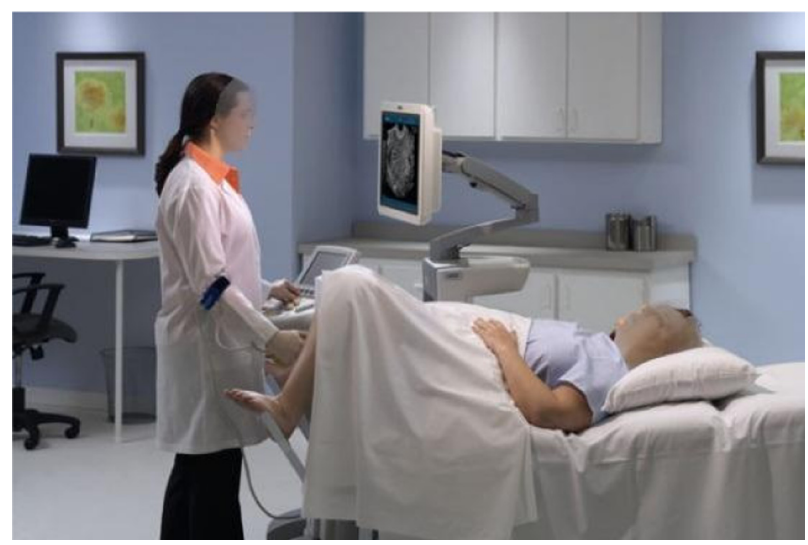

Figure 12 Endovaginal exam performed on table with stirrups. 


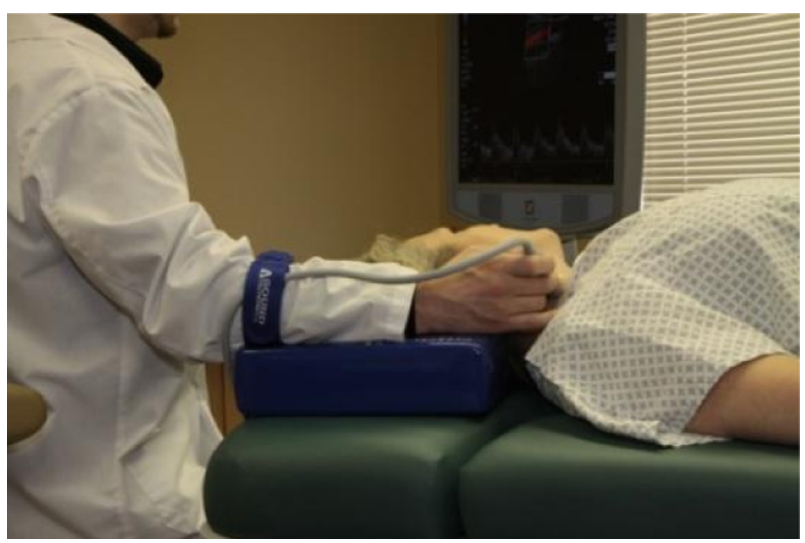

Figure 13 Carotid exam performed from the head of the table.

sonographer to be close to the patient and to position the ultrasound system close; it also gives the sonographer the opportunity to rest his or her forearm on the patient's shoulder.

To reduce abduction and reach when performing bilateral or left lower extremity venous exams, the sonographer should have the patient turn around on the exam table and lie with his/her left leg closest to the sonographer (Figure 15). This will, of course, not be possible with patients who have limited mobility, but the goal is to make work posture changes as often as possible so that awkward and/or static work postures are used only when there is no alternative. Another option for lower extremity exams is to have the patient sit with his/her legs dangling over the edge of the exam table close to the sonographer (Figures 16 and 17). In addition, sonographers should have cooperative patients perform self-augmentation during lower extremity venous exams. By instructing the patient to flex his/her foot toward the knee and then release the flex, the sonographers can eliminate the need to reach to compress the patient's calf (Figure 18). This also eliminates neck and trunk twist.

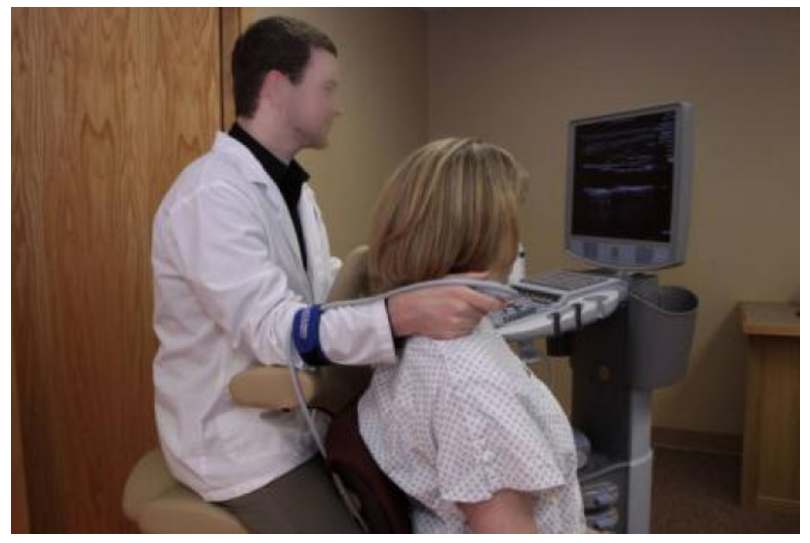

Figure 14 Carotid or thyroid exam performed with patient seated and scanning arm supported.

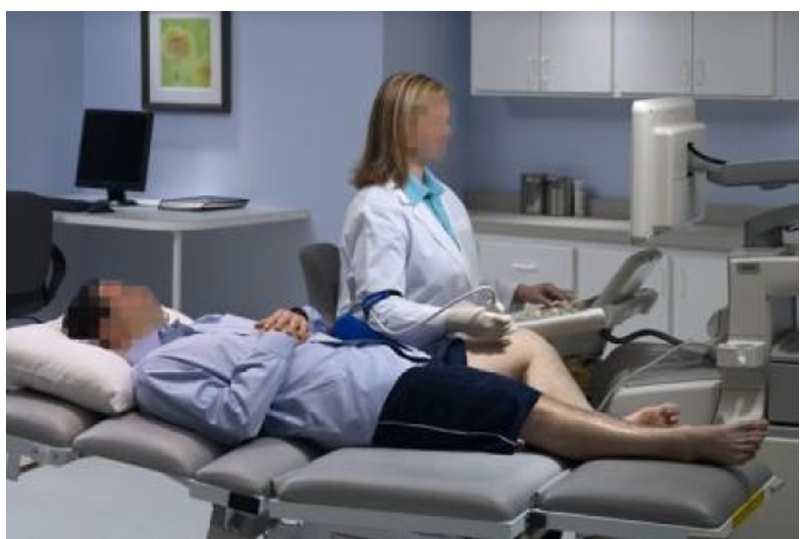

Figure 15 Bilateral lower extremity exam with patient repositioned for left leg.

Ideally, this exam should be performed on an exam table that has a steep angle of reverse Trendelenburg and is raised up enough to allow the sonographers to sit on a chair or stool close to the patient. The ultrasound system's control panel should also have a wide height range so that it can be adjusted low enough to prevent the need to reach up to the controls.

Venous reflux studies can be more comfortably performed by the sonographer with the patient lying on an exam table that achieves an angle of reverse Trendelenberg of at least $40^{\circ}$. The sonographer should then position himself/ herself at the foot of the exam table, sitting or standing at a height that reduces arm abduction and reach (Figure 19). In addition, an ultrasound system with a height-adjustable control panel that can achieve a minimum height of 24 inches reduces the need to reach up to access the controls and reduces neck extension when viewing the monitor (Figures 20 and 21).

The suggested work position for cardiac ultrasound exams is to scan left-handed on the left side of the patient. However, for those sonographers who prefer to scan right-handed, they

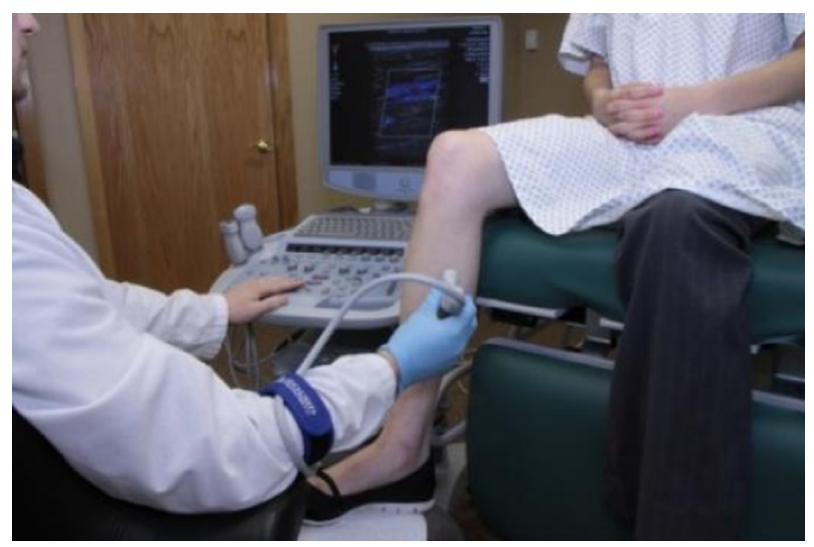

Figure 16 Scanning the lower extremity with patient seated on the exam table. 


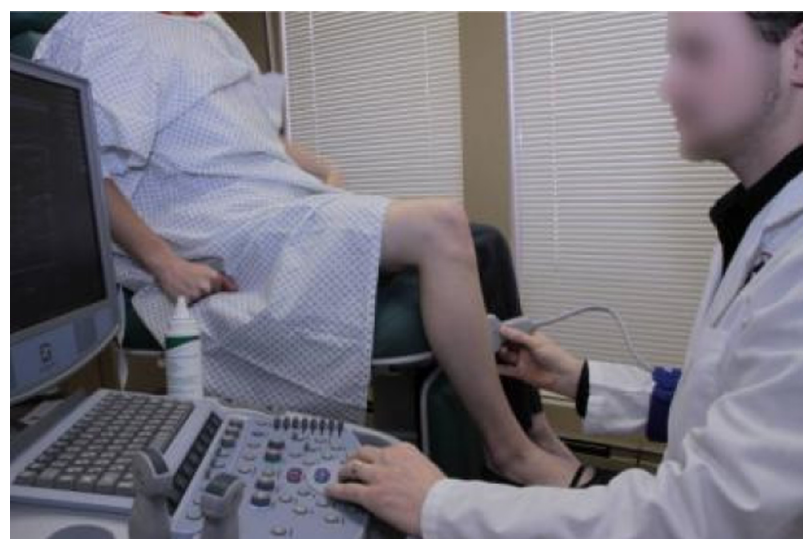

Figure 17 Scanning the lower extremity with patient seated on the exam table.

should try to perform the exam from the left side of the patient but facing the foot end of the exam table (Figure 22). This requires that the ultrasound system be moved from the head of the table to the foot; this can be difficult to achieve if the exam room is not large enough to allow for repositioning of the equipment.

Making changes in established work postures is often difficult and takes some practice but is worth the effort it takes. Implementing small changes over time is often easier. Not all exams can be performed every time with optimal work postures. However, the more often that sonographers can use neutral work postures throughout their workday, the lower their risk for WRMSDs.

\section{Environmental changes}

Although changes in work practices are pivotal in addressing injury risk, administrative support and biomechanical/ environmental changes are also important components. Department managers should establish a work environment in which sonographers feel comfortable reporting injuries

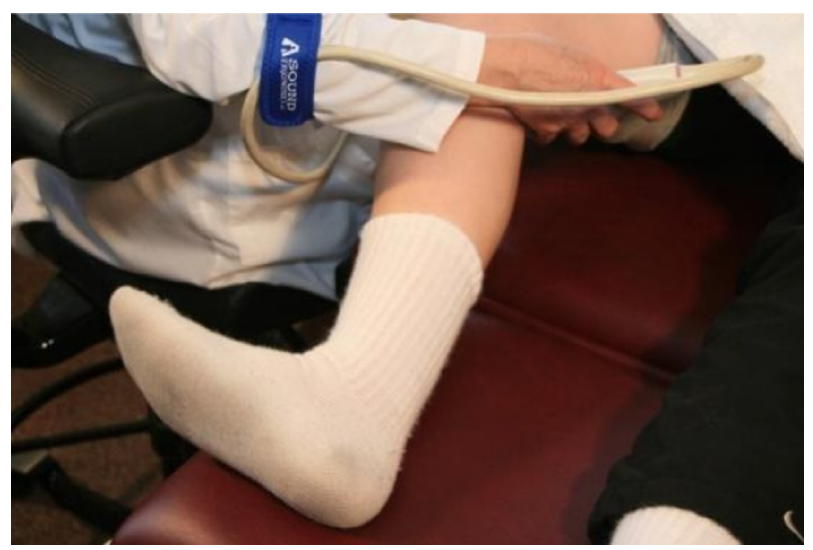

Figure 18 Patient performing self-augmentation.

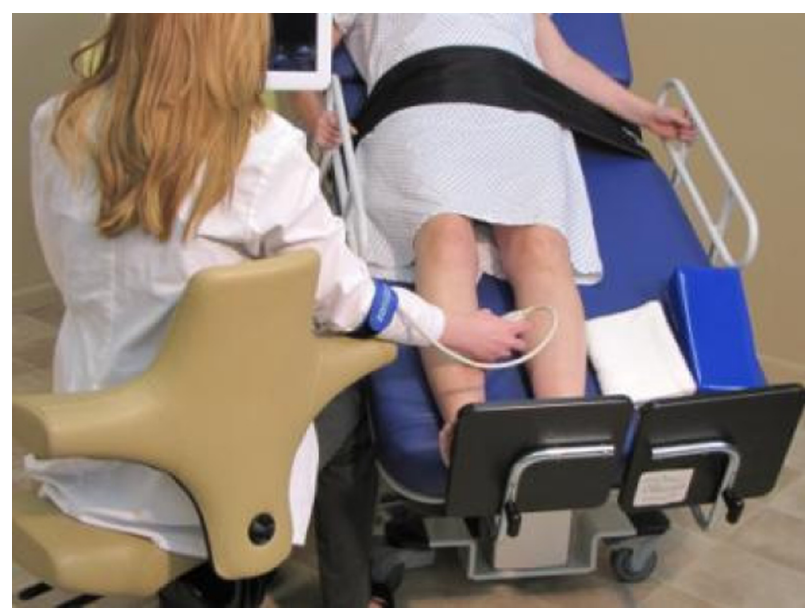

Figure 19 Seated venous reflux exam.

and addressing risk hazards. Managers should be receptive to changing work schedules so that sonographers rotate through a variety of exams and are not repeatedly exposed to high-risk exams without some muscle recovery time. Patient exams should be scheduled with enough time for sonographers to optimize the exam room set up for each exam.

The desire to provide patients with a positive experience should be balanced with the need to provide quality

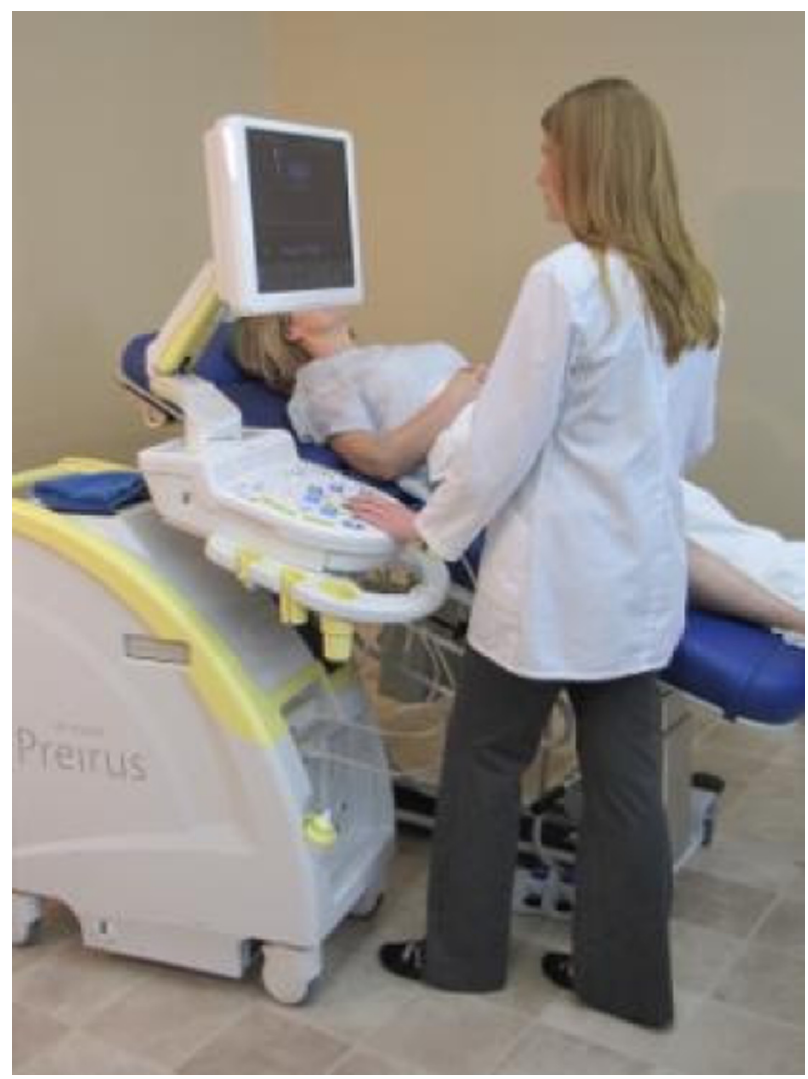

Figure 20 Standing venous reflux exam with adjustable control panel. 


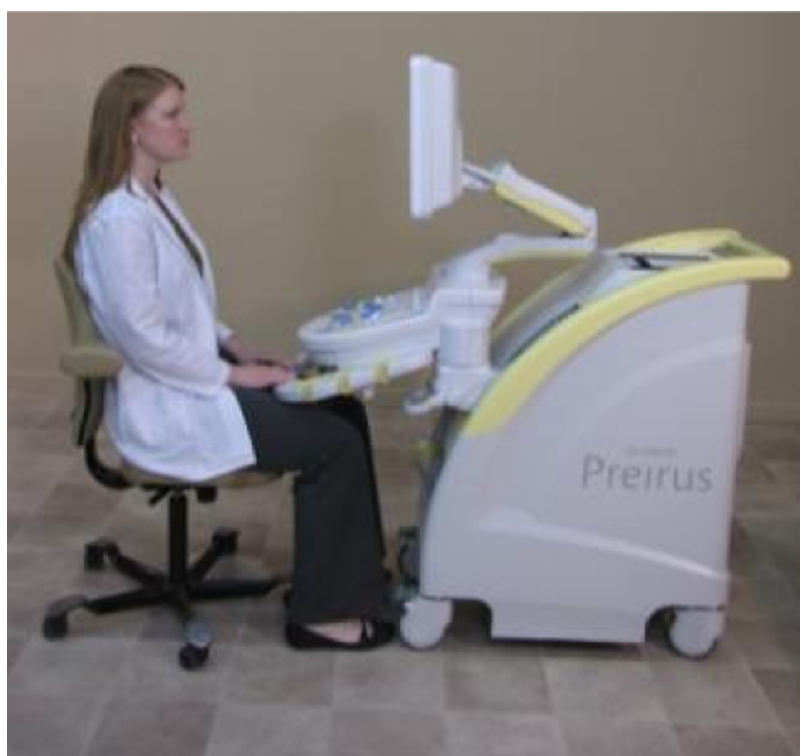

Figure 21 Adjustable control panel at the minimum height.

diagnostic exams while still maintaining a level of worker comfort. This is of particular concern with inpatients and bedside exams. In an effort to prevent patients from having to travel to the ultrasound department or to accommodate the nurses' schedules, many ultrasound exams are needlessly performed at the bedside. These can be among the most injury-producing exams for sonographers, as it is not easy to optimize equipment placement in the patient's room or to move the patient into an accessible position. This results in sonographers having to bend and/or twist to reach the patient throughout the exam. Although these exams should be reserved only for those patients whose health would be compromised if they were to be transported to the ultrasound department, the growing trend of providing all

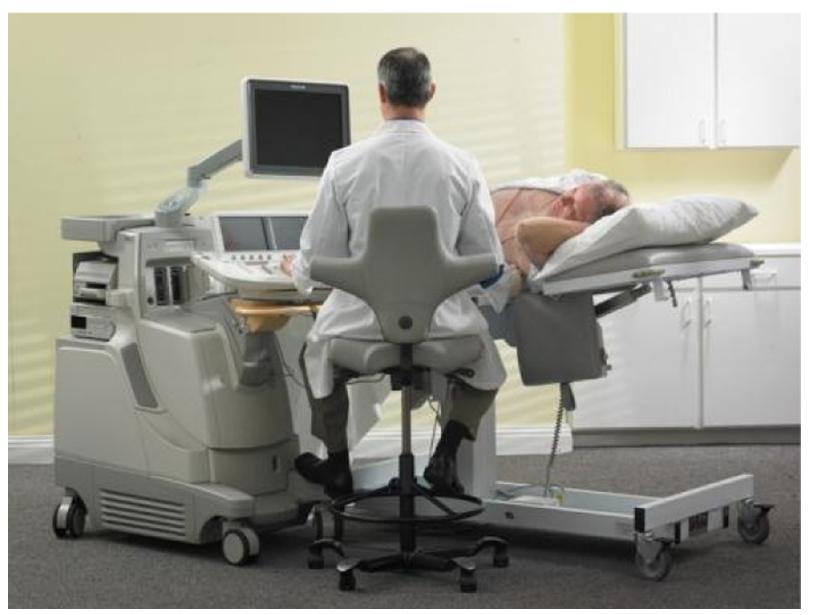

Figure 22 Performing right-handed cardiac exam from the left side of the exam table. patient services at the bedside has created the need to find innovative ways for sonographers to work in more neutral and comfortable positions during these exams.

Managers should support the inclusion of ergonomic features when purchasing new equipment, and if a significant number of bedside exams are performed, the department should have a lightweight, easily movable ultrasound system for those exams. Also, sonographers should have access to ergonomic seating on each patient floor.

Implementing an ergonomics compliance program is one way to demonstrate support for reducing injury risks and to obtain input and feedback from all the workers. Often, this is easier to develop and implement after consulting with an industry-specific ergonomics consultant who can perform a worksite evaluation and develop guidelines that address the needs of each department.

Correct scanning postures are more easily and more consistently achieved when the workstation equipment has multiple ergonomic features. All workstation equipment must be adjustable in order to address the physical dimensions and work style of each sonographer in the department. The ultrasound system should have a monitor that is mounted on an articulated arm so that it can be raised and lowered and so that it can be moved into multiple viewing positions. The control panel should have a wide height range so that sonographers can perform exams seated or standing. The most frequently used controls should be clustered together, and sonographers should position themselves in front of those controls. The system should be easily movable so that it can be positioned close to the exam table (Figures 23 and 24).

The exam table should be height adjustable, ideally by electric controls rather than manual. It should go low enough to accommodate wheelchair transfers and to perform lower extremity exams and high enough to allow sonographers of various heights to stand during exams. Other features can be added to the exam table to enhance its ergonomics. Some of those features might include a dropping foot board and stirrups for pelvic ultrasound exams, a right-side back access panel that allows the sonographer to roll the patient on to his/her left side and step in closer to the patient, a removable left panel that is used to access the apex of the heart during cardiac exams, a detachable headrest for access to the patient's neck, and a detachable arm board for upper extremity exams. And, finally, the exam room chair should have an adequate height range to accommodate the position of the table throughout each exam. The chair should have a seat pan that promotes the natural curvature of the spine and should be easy to adjust from a seated position. All exam 


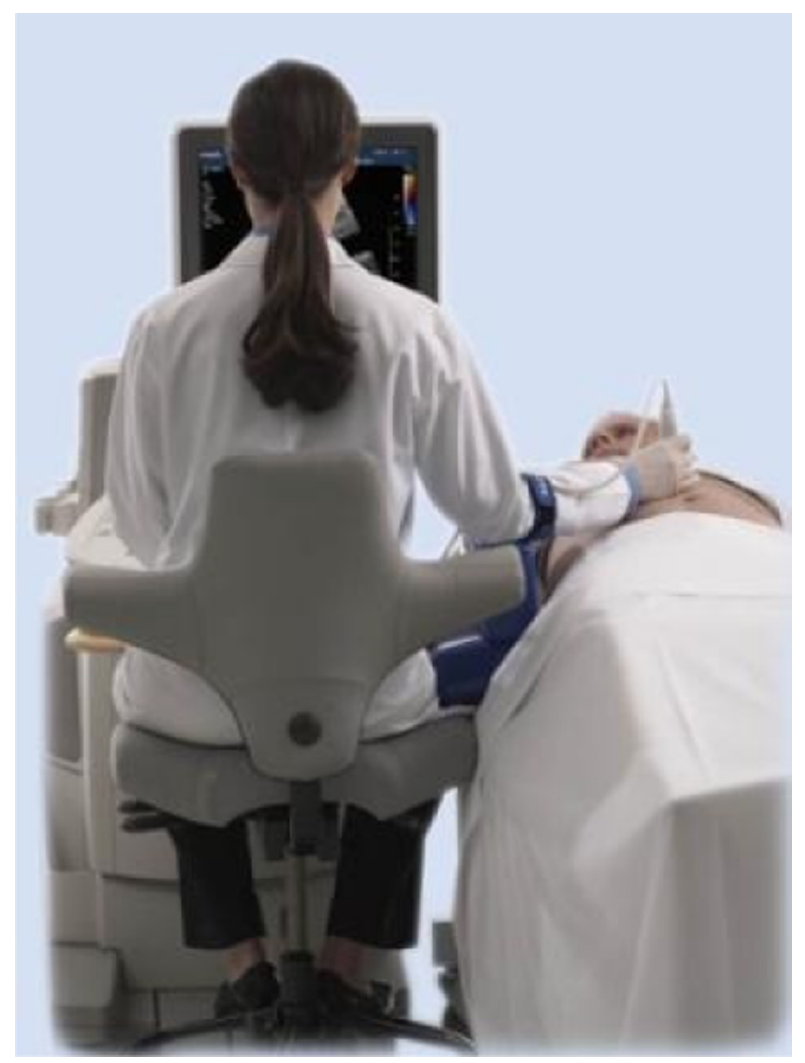

Figure 23 Correct scanning posture.

room equipment should be quick and easy to adjust; otherwise, sonographers will not take the time to optimize the features of the equipment.

The ultrasound work environment also includes the image archiving computer workstation and the reporting computer workstation. Computer desks should be height adjustable and desk chairs should have lumbar support, height adjustability, and features different from those of

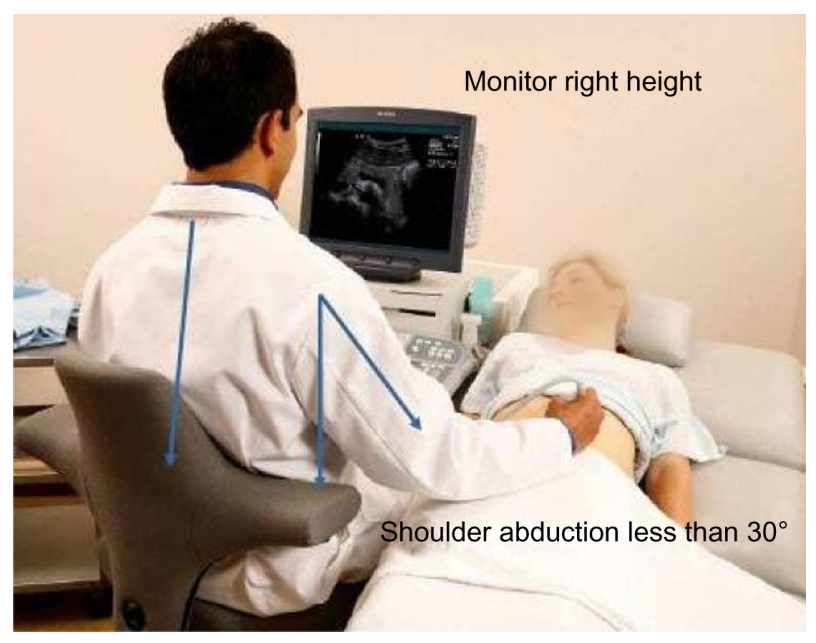

Figure 24 Correct scanning posture.

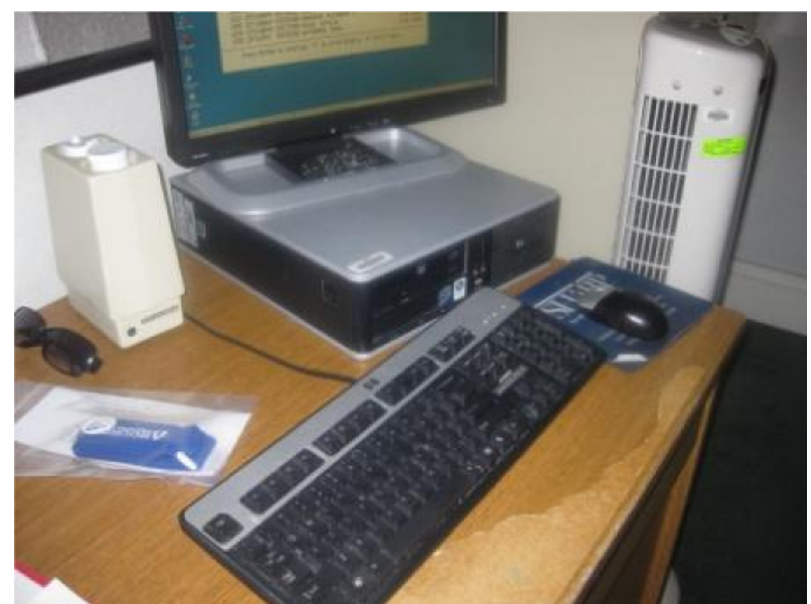

Figure 25 Monitor is occupying desktop space and is positioned off-center to viewer.

exam room chairs. The computer monitors should be wall mounted on articulated arms for multiple viewing positions and to free up desk space. They should also be positioned directly in front of the user (Figure 25). Computer towers should not be positioned under the desks so that they interfere with worker leg positioning (Figure 26). The keyboard and mouse should be positioned so that excessive reaching is unnecessary (Figures 27 and 28). Shelves and other storage areas should be located so that reaching is minimized.

\section{Summary}

The following is a summary of the best practices for reducing the risk for WRMSDs in sonography.

- Position the patient close to the sonographer.

- Try to perform some exams from the head or foot of the exam table; try some exams with the patient seated.

- Use a palmar transducer grip whenever possible.

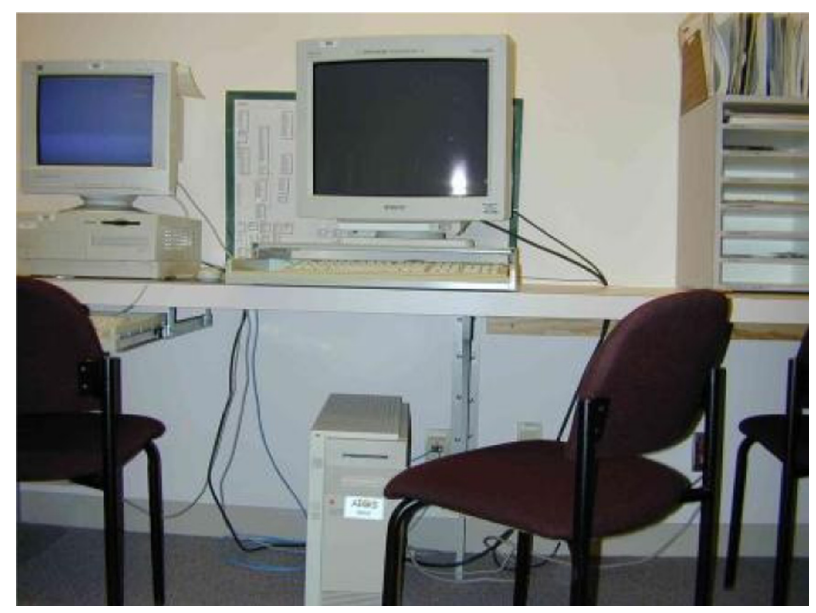

Figure $\mathbf{2 6}$ Computer tower is obstructing leg position and access to the desk. 


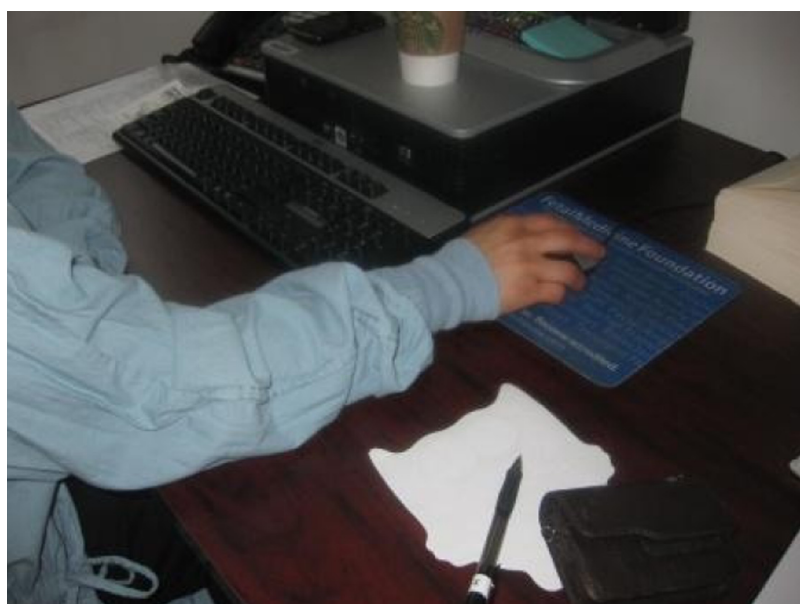

Figure 27 Reaching for mouse.

- Try different transducer grips throughout exams in order to maintain a neutral wrist position.

- Utilize the ergonomic features of the exam room equipment in order to reduce arm abduction, reaching, and neck and trunk twisting.

- Have cooperative patients self-augment venous flow during lower extremity exams.

- Utilize breaks for muscle recovery.

- Optimize the computer workstation equipment for more comfortable positions.

- Avoid unnecessary bedside exams.

- Avoid performing the same type of exam repeatedly throughout the workday.

- Consider the ergonomic features when purchasing new equipment.

- Above all, avoid static postures. It is important to incorporate movement into work activities.

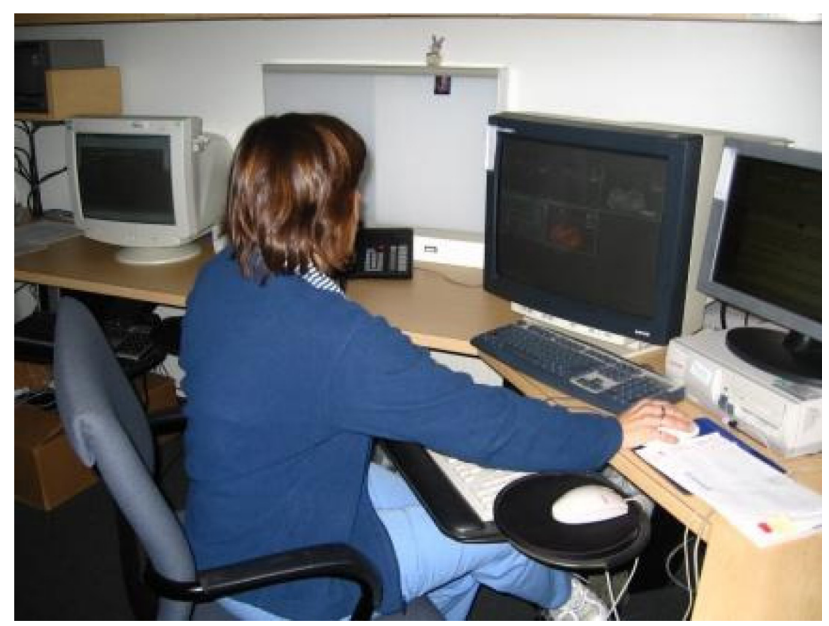

Figure 28 Reaching for keyboard.

\section{Conclusion}

WRMSDs among sonographers can impact the quality of patient care, can have a financial impact on both the ultrasound department and the individual worker, and can negatively affect the morale of the workplace. The multiple causes of these injuries require multiple approaches to their prevention. At the center of any injury prevention program, however, is the participation of the worker. Sonographers must recognize injury risk hazards, utilize the ergonomic features of their workstation equipment, and be willing to make changes in their work postures. The administrative environment of the sonography workplace must support these attempts to change and to report injuries. Without this synergy, it is difficult to effectively reduce the incidence of workplace injuries.

\section{Disclosure}

The author declares no conflicts of interest in this work.

\section{References}

1. Melhorn JM. Cumulative trauma disorders and repetitive strain injuries. The future. Clin Orthop.1998;351:107-126

2. Craig M. Sonography: an occupational health hazard? J Diagn Med Sonogr. 1985;1:121-125.

3. Vanderpool HE, Friis EA, Smith BS, Harms KL. Prevalence of carpal tunnel syndrome and other work-related musculoskeletal problems in cardiac sonographers. J Occup Med. 1993;35:604-610.

4. Pike I, Russo A, Berkowitz J, Baker J, Lessoway V. The prevalence of musculoskeletal disorders among diagnostic medical sonographers; J Diagn Med Sonogr. 1997;13(5):219-227.

5. Evans K, Roll S, Baker J. Work-related musculoskeletal disorders (WRMSD) among registered diagnostic medical sonographers and vascular technologists: a representative sample. J Diagn Med Sonogr. 2009;9;25(6):287-299.

6. Necas M. Musculoskeletal symptomatology and repetitive strain injury in diagnostic medical sonographers. A pilot study in Washington and Oregon. J Diagn Med Sonogr. 1996;12(6):266-273.

7. Faragher EB, Cass M, Cooper CL. The relationship between job satisfaction and health: a meta analysis. Occup Environ Med. 2005;62:105-1128.

8. Centers for Disease Control and Prevention. Adult obesity facts Available from: http://www.cdc.gov/obesity/data/adult.html. Accessed November 1, 2013.

9. Lindle RS, Metter EJ, Lynch NA, et al. Age and gender comparisons of muscle strength in 654 women and men aged 20-93 yr. J Appl Physiol. 1997;83(5):1581-1587.

10. Occupational Safety and Health Administration. https:/www.osha.gov/ SLTC/etools/hospital/sonography/sonography.html.

11. Evans KD, Roll SC, Hutmire C, Baker JP. Factors that contribute to wrist-hand-finger discomfort in diagnostic medical sonographers and vascular technologists J Diagn Med Sonogr. 2010;26(3):121-129.

12. Russo A, Murphy C, Lessoway V, Berkowitz J. The prevalence of musculoskeletal symptoms among British Columbia sonographers. Appl Ergon. 2002;33(5):385-393.

13. Roll SC, Evans KD, Hutmire CD, Baker JP. An analysis of occupational factors related to shoulder discomfort in diagnostic medical sonographers and vascular technologists. Work. 2012;42(3):355-365.

14. Rempel DM, Harrison RJ, Barnhart S. Work-related cumulative trauma disorders of the upper extremity. JAMA. 1992;267(6):838-842. 
15. US Department of Health and Human Services (DHHS), National Institute for Occupational Safety and Health (NIOSH). Preventing work-related musculoskeletal disorders in sonography. Publication No 2006-148; 2006.

16. De Krom MC, Kester AD, Knipschild PG, Spaans F. Risk factors for carpal tunnel syndrome. Am J Epidemiol. 1990;132(6):1102-1110.

17. Kroemer KHE, Grandjean E. Fitting the Task to the Human, 5th ed. 1998.

18. Village J, Trask C. Ergonomic analysis of postural and muscular loads to diagnostic sonographers. Int J Ind Ergon. 2007;37(9-10):781.

19. Vedsted P, Blangsted AK, Soggard K, Orizio C, Sjogaard G. Muscle tissue oxygenation, pressure, electrical and mechanical responses during dynamic and static voluntary contractions. Eur J Appl Physiol. 2006;96:165-177.
20. Nussbaum MA. Static and dynamic myoelectric measures of shoulder muscle fatigue during intermittent dynamic exertions of the low to moderate intensity. Eur J Appl Physiol. 2001;85:299-309.

21. Pennett L, Fine LJ, Keyserling WM, Herrin GD, Chaffin DB. Shoulder disorders and postural stress in automobile assembly work. Scand $J$ Work Environ Health. 2000;25(4):283-291.

22. Hill JJ 3rd, Slade MD, Russi M. Anthropometric measurements, job characteristics and prevalence of musculoskeletal symptoms in female medical sonographers. Work. 2009;33:181-189.

23. Coffin CT. The use of a vertical arm support device to reduce upper extremity muscle firing in sonographers. Work. 2012;42(3): $367-371$

24. Murphey S, Milkowski A. Surface EMG evaluation of sonographer scanning postures. J Diagn Med Sonogr. 2006;22(5):298-205.

Reports in Medical Imaging

\section{Publish your work in this journal}

Reports in Medical Imaging is an international, peer-reviewed, open access journal publishing original research, reports, reviews and commentaries on all areas of medical imaging. The manuscript management system is completely online and includes a very quick and fair peer-review system, which is all easy to use.

\section{Dovepress}

Visit http://www.dovepress.com/testimonials.php to read real quotes from published authors. 